IPMU 09-0133

ICRR-Report-554-2009-16

\title{
Probing the primordial power spectra with inflationary priors
}

\author{
Masahiro Kawasaki ${ }^{1,2}$, Toyokazu Sekiguchi ${ }^{1}$ \\ ${ }^{1}$ Institute for Cosmic Ray Research, University of Tokyo, Kashiwa 277-8582, Japan \\ ${ }^{2}$ Institute for the Physics and Mathematics of the Universe, University of Tokyo, \\ Kashiwa, Chiba, 277-8568, Japan
}

\begin{abstract}
We investigate constraints on power spectra of the primordial curvature and tensor perturbations with priors based on single-field slow-roll inflation models. The Hubble slow-roll parameters are included in cosmological parameters and the primordial power spectra are generated using the inflationary flow equations. Using data from recent observations of $\mathrm{CMB}$ and several measurements of geometrical distances in the late Universe, we perform Bayesian parameter estimation and model selection for models that have separate priors on the slow-roll parameters. The same analysis is also performed adopting the standard parameterization of the primordial power spectra. We confirmed that the scale-invariant Harrison-Zel'dovich spectrum is disfavored with more significance than previous studies. While current observations appear to be optimally modeled with some simple models of single-field slow-roll inflation, data is not enough constraining to distinguish these models.
\end{abstract}




\section{Introduction}

Cosmological observations, including the cosmic microwave background (CMB), large scale structure, baryon acoustic oscillation (BAO), type Ia supernovae (SN) offer opportunities to probe new physics far beyond the reach of experiments in terrestrial laboratories. One of such physics is inflation, that solves various problems in the hot universe scenario [1 5]. In addition, inflation also explains generation of initial perturbations for structure formation [6 13]. At present, Inflation is an essential part of our best description of the Universe.

The simplest class of models of inflation is so-called single-field slow-roll inflation [4,5], where potential energy of a single scalar field (inflaton), whose field value varies slowly, drives an exponential expansion of the Universe. Gaussian, nearly scale-invariant primordial curvature perturbation can be generated from the vacuum fluctuation of inflaton in a quasi-de Sitter spacetime. Such primordial perturbation gives excellent fits to various data from cosmological observations, which makes single-field slow-roll inflation highly attractive. However we yet know quite little about inflaton and its potential.

Identification of inflaton is of particular interest both in cosmology and particle physics. As a model of single-field slow-roll inflation left its vestige in the late Universe in an observable way through generation of the primordial curvature and tensor perturbations, constraints on models have been mainly investigated through constraining power spectra of these primordial perturbations, $\mathcal{P}_{\zeta}(k)$ and $\mathcal{P}_{h}(k)$, using various cosmological observation \#1. One of the most familiar ways may be to adopt the standard parameterization for the power spectra, $A_{s}, r, n_{s}, n_{t}, \alpha_{s}$, etc. (See Eqs. (15) 19)), and derive constraints on these parameters. Some more involved analyses have also been performed focusing on reconstruction of the potential of the inflaton or slow-roll flow parameters [14 32 . In either way, deviation from the Harrison-Zel'dovich (HZ) spectrum (i.e. $r=0$ and $n_{s}=\alpha_{s}=\ldots=0$ ) allows us to probe models of single-field slow-roll inflation. Cosmological data now signifies some spectral features in the power spectrum of the curvature perturbation. When we assume a power-law for curvature perturbation spectrum $\mathcal{P}_{\zeta}(k) \propto A_{s} k^{n_{s}-1}$ and absence of the tensor perturbation $\mathcal{P}_{h}(k)=0$, recent CMB data from WMAP give $-0.065<n_{s}-1<-0.009$ (95\% C.L.), suggesting a significant deviation from the HZ power spectrum [33].

However, constraints on the primordial power spectra are highly dependent on parameter spaces which we investigate. For instance, if running of the spectral index $\alpha_{s}$ is included, the same data allow relatively large $\alpha_{s}$ [33]. This kind of issues commonly arise when we try to constrain the primordial power spectra in parametric ways. Consequently, shapes of reconstructed power spectra also differ depending on the choice of parameter spaces, which finally affects selection among inflation models.

Since we do not in advance know a parameter space where we should explore a constraint on the power spectrum, we also need to examine whether the parameter space

\#1 There are also several other probes for inflation models, including primordial isocurvature perturbations and non-Gaussianity in primordial perturbations. 
is appropriate. More generally, appropriateness of a model, which possesses its own prior assumption, should also be discussed. A guiding principle in looking for an optimal model is Occam's razor, which penalizes unnecessary assumption in describing observations. Bayesian model selection is Bayesian implementation of Occam's razor, which is now frequently applied in the context of cosmology 34 52. In particular, the authors in Ref. [25] have adopted Bayesian model selection to assess optimal orders up to which reconstruction of inflaton potential should be performed. Moreover, in Ref. [53] Bayesian model selection is directly applied to distinguish some class of inflation models.

Motivated by [25, 53] and other earlier studies, in this paper we investigate an optimal constraint on primordial perturbation spectra and make comparison of single-field slowroll inflation models using recent cosmological observations, which would be a subject of great interest [54]. For these purpose we make vigorous use of Bayesian model selection. We compare Bayes evidences for several models which have separate priors on inflationary slow-roll parameters, or parameters of primordial power spectra. Each of these models can be regarded as representing some class of single-field slow-roll inflation models. In this paper we implicitly assume a Freedman-Robertson-Walker universe and adopt natural units $\hbar=c=M_{\mathrm{Pl}}=1$.

This paper is organized as follows: In Section 2 we briefly review the Hubble slow-roll flow equations, which are adopted in our analysis. Then in Section 3, some essences of Bayesian model selection are also reviewed. The main part of our paper is Section 4, where we investigate constraints on the primordial power spectra using data from recent observations of $\mathrm{CMB}$ and geometrical distances in the late Universe. By employing Bayesian model selection, an optimal constraint and comparison of models of single-field slow-roll inflation are investigated. The final section is devoted to summary and future outlook.

\section{Hubble slow-roll flow equations}

We make use of the Hubble slow-roll (HSR) flow equations [16, 19, 55], a specific version of more general inflationary flow equations [56, 57]. Our algorithm is basically identical to slow-roll reconstruction presented in [23, 30] and we here quote some key consequences. In this paper, we assume models of single-field slow-roll inflation have canonical kinetic terms and do not investigate non-canonical models.

During an epoch of slow-roll inflation, the inflaton field $\phi$ can be safely assumed as a monotonic function of the time and hence regarded as a generalized time coordinate. Instead of solving the Hamilton-Jacobi equation [57] for given inflaton potential, we rather solve a set of the HSR flow equations given by

$$
\begin{aligned}
\epsilon_{H}(\phi) & =\frac{1}{4 \pi}\left(\frac{H(\phi)^{\prime}}{H(\phi)}\right)^{2} \\
{ }^{\ell} \lambda_{H}(\phi) & =\left(\frac{1}{4 \pi}\right)^{\ell} \frac{\left(H(\phi)^{\prime}\right)^{\ell-1}}{H(\phi)^{\ell}} \frac{d^{\ell+1} H(\phi)}{d \phi^{\ell+1}} \quad(\text { for } \ell \geq 1) .
\end{aligned}
$$


Here and hereafter we denote derivatives respective to $\phi$ as prime (e.g. $H^{\prime} \equiv d H / d \phi$ ). Notice that $\phi$ plays a role of time variable in this formalism. These flow equations are solved once we specify the initial values for $\epsilon_{H}$ and ${ }^{\ell} \lambda_{H}$ at a fiducial $\phi_{*}$,

$$
\begin{aligned}
\epsilon_{H *} & \equiv \epsilon_{H}\left(\phi_{*}\right), \\
{ }^{\ell} \lambda_{H *} & \equiv{ }^{\ell} \lambda_{H}\left(\phi_{*}\right) \quad(\text { for } \ell \geq 1) .
\end{aligned}
$$

If we choose fiducial HSR parameters ${ }^{\ell} \lambda_{H *}=0$ for $\ell>M$, the HSR parameters ${ }^{\ell} \lambda_{H}(\phi)$ for $\ell>M$ also vanish at any $\phi$ and hence the flow equations are truncated at order $M$. In this case, the Hubble expansion rate can be exactly solved [58]. Without loss of generality, we can choose $\phi_{*}=0$ and $\phi$ being a decreasing function of time (i.e. $H^{\prime}(\phi)>0$ ). Then we obtain

$$
H(\phi)=H_{*}\left(1+B_{1} \phi+B_{2} \phi^{2}+\cdots B_{M+1} \phi^{M+1}\right),
$$

where $H_{*}=H\left(\phi_{*}\right)=H(0)$ and the coefficients of the Taylor expansion, $B_{i}(i=1, \ldots, M+$ $1)$, are given by the HSR parameters at the fiducial point,

$$
\begin{aligned}
B_{1} & =\sqrt{4 \pi \epsilon_{H *}}, \\
B_{\ell+1} & =\frac{(4 \pi)^{\ell}}{(\ell+1) ! B_{1}^{\ell-1}}{ }^{\ell} \lambda_{H *} \quad(\text { for } \ell \geq 1) .
\end{aligned}
$$

Since we have specified the function $H(\phi)$, we already know the dynamics of the background universe and related variables as functions of $\phi$. For example, e-folding number $N(\phi)$ at time $\phi$ is given by integrating

$$
\frac{d N}{d \phi}=-\sqrt{\frac{4 \pi}{\epsilon_{H}(\phi)}} .
$$

Similarly, a wave number that exits the horizon at time $\phi$ is given by integrating

$$
\frac{d \ln k}{d \phi}=-\sqrt{\frac{4 \pi}{\epsilon_{H}(\phi)}}\left(1-\epsilon_{H}(\phi)\right) .
$$

Once we fix a fiducial wave number $k_{*}=k\left(\phi_{*}\right)$, there is a one-to-one correspondence in $k$ and $\phi$ by Eq. (9), as long as slow roll of the inflaton does not break down. Then any other function of $\phi$ is rewritten as that of $k$. For later convenience, we choose the efolding number $N(k)$ so that it vanishes at a wave number $k=10^{-4} \mathrm{Mpc}^{-1}$, which roughly corresponds to the scale of current horizon $\simeq 10 \mathrm{Gpc}$.

In this paper, we truncate the HSR flow equations at $M=2$. This is because higher order HSR parameters may not be important due to the limited range of observable wave numbers $\mathcal{O}\left(10^{-4}\right)<k<\mathcal{O}(0.1) \mathrm{Mpc}^{-1}$ and insufficient accuracy of data at present or in 
the near future. The fiducial HSR parameters up to $M=2$ are nothing but the usual slow-roll parameters $\epsilon(\phi), \eta(\phi)$ and $\xi(\phi)$ and they are given by

$$
\begin{aligned}
\epsilon(\phi) & =\epsilon_{H}(\phi), \\
\eta(\phi) & ={ }^{1} \lambda_{H}(\phi), \\
\xi(\phi) & ={ }^{2} \lambda_{H}(\phi) .
\end{aligned}
$$

One of the most important prediction of single-field inflation models is that the primordial curvature and tensor perturbations are not independent of but related to each other since both are generated from the dynamics of the single scalar field $\phi$. Thus there exists the consistency relation for single-field inflation models. At second order in slow-roll approximation, the power spectrum of the primordial curvature and tensor perturbations, $\mathcal{P}_{\zeta}(k)$ and $\mathcal{P}_{h}(k)$, respectively, are given by [59]

$$
\begin{aligned}
& \mathcal{P}_{\zeta}(k)=\left.\frac{[1-(2 C+1) \epsilon(\phi)+C \eta(\phi)]^{2}}{\pi \epsilon(\phi)} H(\phi)^{2}\right|_{\phi=\phi(k)}, \\
& \mathcal{P}_{h}(k)=\left.\frac{16[1-(C+1) \epsilon(\phi)]^{2}}{\pi} H(\phi)^{2}\right|_{\phi=\phi(k)},
\end{aligned}
$$

where $C=-2+\ln 2+\gamma \approx-0.729637$ and $\gamma$ is the Euler constant. Although Eqs. (13) 14) are originally derived assuming $\epsilon$ and $\eta$ are constants [59], evolution of these variables are practically very small at observable scales $k$, which validates the use of Eqs. (13, 14, \#2.

The standard parameters of the primordial power spectra are given by Taylor expanding the logarithm of Eqs. (13, 14),

$$
\begin{aligned}
A_{s} & \equiv P_{\zeta}\left(k_{*}\right), \\
n_{s} & \left.\equiv \frac{d \ln \mathcal{P}_{\zeta}}{d \ln k}\right|_{k=k_{*}}+1=1+2 \eta_{*}-4 \epsilon_{*}-2(1+\mathcal{C}) \epsilon_{*}^{2}-\frac{3-5 \mathcal{C}}{2} \epsilon_{*} \eta_{*}+\frac{3-\mathcal{C}}{2} \xi_{*}, \\
r & \left.\equiv \frac{P_{h}}{P_{\zeta}}\right|_{k=k_{*}}=16 \epsilon_{*}\left(1+2 C\left(\epsilon_{*}-\eta_{*}\right)\right), \\
\alpha_{s} & \left.\equiv \frac{d^{2} \ln \mathcal{P}_{\zeta}}{d \ln k^{2}}\right|_{k=k_{*}}=-2 \xi_{*}-8 \epsilon_{*}^{2}+10 \epsilon_{*} \eta_{*}, \\
n_{t} & \left.\equiv \frac{d \ln \mathcal{P}_{h}}{d \ln k}\right|_{k=k_{*}}=-2 \epsilon_{*}-(3+\mathcal{C}) \epsilon_{*}^{2}+(1+\mathcal{C}) \epsilon_{*} \eta_{*},
\end{aligned}
$$

where $\mathcal{C}=4(\ln 2+\gamma)-5$. With these standard parameters, we can approximate the power

\#2 Several authors [29, 30, have discussed effects of differences in the power spectra calculated from exact solution of the wave equation, approximation with slow-roll parameters up to the 2nd order (i.e. Eqs. (13, 14) ). They conclude that differences among the exact and these approximated power spectra are not significant with a certain prior on the e-folding number $N$. 
spectra by

$$
\begin{aligned}
& \mathcal{P}_{\zeta}(k)=A_{s} \exp \left[\left(n_{s}-1\right) \ln \frac{k}{k_{*}}+\frac{1}{2} \alpha_{s}\left(\ln \frac{k}{k_{*}}\right)^{2}\right], \\
& \mathcal{P}_{h}(k)=r A_{s} \exp \left[n_{t} \ln \frac{k}{k_{*}}\right] .
\end{aligned}
$$

Along with the power spectra in Eqs. (13,-14), the duration of the inflation is also important. We impose a prior on the e-folding number, $N>25$. This prior roughly means that the energy scale of inflation should be higher than $\mathrm{TeV}[23] \#$. Imposition of this prior ensures that inflaton does not affect physics below electroweak scales. However, this does not necessarily mean that a given model of single-field slow-roll inflation with certain $\left(\epsilon_{*}, \eta_{*}, \xi_{*}\right)$ is excluded if it predicts $N<25$, for slow-roll parameters at higher orders can maintain slow-rolling of the inflaton further. Thus we cannot strictly restrict models of single-field slow-roll inflation with a prior $N>25$. In Section 4, as default, we adopt a prior $N>25$ in investigating constraints on the primordial power spectra with inflationary priors. However, we also investigate constraints without the prior $N>25$, which would be informative in discussing what are plausible models supported from data.

\section{Bayesian model selection}

As we have mentioned in Introduction, we adopt Bayesian model selection in order to compare different models of single-field slow-roll inflation. Before presenting details of our analysis, let us briefly review some essences of Bayesian model selection. We also refer to [46, 47, 49] for more detailed reviews.

Given data, a joint posterior distribution for a model $M$ and its model parameter $\Theta$, $P(\Theta, M \mid$ data $)$, is given by the hierarchical Bayes theorem,

$$
P(\Theta, M \mid \text { data })=\frac{P(\text { data } \mid \Theta, M) P(\Theta \mid M) P(M)}{P(\text { data })},
$$

where $P($ data $\mid \Theta, M)=\mathcal{L}(\Theta)$ is the likelihood function, $P(\Theta \mid M)$ is the prior distribution for $\Theta$ specified by $M$, and $P(M)$ is the prior distribution for $M$. The remaining $P$ (data) is an irrelevant normalization constant. Then the posterior distribution for $M, P(M \mid$ data $)$ is given by marginalizing $P(\Theta, M \mid$ data $)$ over the model parameters $\Theta$,

$$
\begin{aligned}
P(M \mid \text { data }) & =\int d \Theta P(\Theta, M \mid \text { data }) \\
& =\frac{P(M)}{P(\text { data })} \int d \Theta P(\text { data } \mid \Theta, M) P(\Theta \mid M)
\end{aligned}
$$

\footnotetext{
\#3 The role of a prior on the e-folding number is detailedly discussed in [60].
} 
The final integral in Eq. (23) is called Bayes evidence $E(M)$,

$$
E(M) \equiv \int d \Theta P(\text { data } \mid \Theta, M) P(\Theta \mid M),
$$

which measures marginalized likelihood of the model $M$. Thus the relative likelihood of different models $M_{i}$ and $M_{j}$ is assessed by the ratio of their Bayes evidences,

$$
B_{i j} \equiv \ln \frac{E\left(M_{i}\right)}{E\left(M_{j}\right)}
$$

which is called Bayes factor. Bayesian model selection is implemented by estimating Bayes factor which measures relative likelihood between two different models. Jeffreys' scale is often adopted to connect numbers and semantics, which states: for $\left|B_{i j}\right|<1$ the evidence is not significant; $1<\left|B_{i j}\right|<2.5$ significant; $2.5<\left|B_{i j}\right|<5$ strong; and $5<\left|B_{i j}\right|$ decisive\#4.

In usual parameter estimation, a posterior distribution of parameters for a model $M$, $P(\Theta \mid$ data, $M)$ is investigated. However, our purpose in this paper of comparing different models of inflation is not achieved via $P(\Theta \mid$ data, $M)$, where a model $M$ is by definition assumed to be true. This is another way of representing our statement in Introduction, that different models cannot be compared with a fixed parameter space. Instead, comparison of different models is achieved by the Bayes factors $B_{i j}$. This is why we adopt the Bayesian model selection in our analysis. By assigning a prior $P(\Theta \mid M)$ to reflect prediction of a class of single-field inflation models $M$, the likelihood of $M$ is measured with the Bayes evidence $E(M)$ and two different classes can be compared.

On the other hand, we can also explore an optimal constraint on parameters $\Theta$ by adopting Bayesian model selection. By comparing different models, which have separate priors on $\Theta$, an optimal constraints can be obtained from a posterior distribution $P(\Theta \mid$ data, $M)$ of the parameters for a model which has higher Bayes evidence than other models.

One remark should be mentioned. The Bayes evidence explicitly depends on a prior probability $P(\Theta \mid M)$ due to its normalization. For example let us consider that some two different top-hat priors are imposed on a same parameter. Even though the parameter region of high-likelihood is sufficiently covered by both of the priors, the resultant Bayes evidences differs, that are inversely proportional to the ranges of the top-hat priors. This is very contrastive to the usual parameter estimation, where the posterior probabilities from these two different priors do not differ much.

\section{Analysis with observational data}

Here we investigate constraints on the primordial power spectra and make comparison of single-field slow-roll inflation models. First of all, since there are quite a large number

\#4 The choice of numbers and semantics is more or less arbitrary and differs literature by literature. For instance, a more conservative statement is suggested in [46]. 
of theoretical models of single-field slow-roll inflation, it is difficult to analyze them individually. Instead we consider several different models which have separate priors on the primordial power spectra. Each of these models can be regarded as representing some class of single-field slow-roll inflation models. We adopt two parameterizations of the primoridial power spectra. One is the standard parameterization of Eqs. (20,21), that has $A_{s}, n_{s}, r$ and $\alpha_{s}$. As we are interested in single-field slow-roll inflation models, we impose the standard inflation consistency relation, $n_{t}=-r / 8$, when we adopt the standard parameterization. The other is the HSR parameterization that has $A_{s}, \epsilon_{*}, \eta_{*}$ and $\xi_{*}$. By adopting these two parametrizations, the models we compare are listed in Table 1. The reference model is $M_{\mathrm{HZ}}$ that has the $\mathrm{HZ}$ primordial power spectrum. $M_{n_{s}}, M_{n_{s} r}$ and $M_{n_{s} \alpha_{s}}$ have different top-hat priors on the standard parameters $n_{s}, r$ and $\alpha_{s}$, that are described in the last column of Table 1. On the other hand, $M_{\epsilon}, M_{\eta}, M_{\eta \xi}$ have different top-hat priors on the HSR parameters $\epsilon_{*}$ and $\eta_{*}$ that are also described in the last column of Table 1 \#5. In investigating models with the HSR parameters, we impose an additional prior $N>25$ as default. Basically, subscripts in names of models represent varied parameters in the model, and other parameters absent in the subscripts are fixed to the default values. The only exception is $\epsilon_{*}$ which is varied in a small range $\left[0,10^{-4}\right]$ in $M_{\eta} \#$. This is because $\epsilon_{*}$ cannot be fixed to zero as the inflaton cannot roll when $\epsilon_{*}=0$. Regarding priors on $A_{s}$, we adopt a common prior $2.5<\ln \left[10^{10} A_{s}\right]<3.5$ in all models. In our analysis a fiducial wave number is chosen to be $k_{*}=0.01 \mathrm{Mpc}^{-1 \# 7}$.

We assume a flat $\Lambda$ CDM model as background cosmology. In addition to parameters representing the shape of the primordial power spectra (i.e. $\left(A_{s}, n_{s}, r, \alpha_{s}\right)$ or $\left(A_{s}, \epsilon_{*}, \eta_{*}\right.$, $\left.\xi_{*}\right)$ ), following parameters are included in the analysis as primary cosmological parameters,

$$
\left(\omega_{b}, \omega_{c}, \theta_{s}, \tau, A_{S Z}\right)
$$

where $\omega_{b}$ and $\omega_{c}$ are the density parameters of baryon and CDM, respectively, $\theta_{s}$ is the angular scale of the acoustic horizon [62, $\tau$ is the optical depth of reionization, and $A_{S Z}$ is the amplitude of template Sunyaev-Zel'dovich power spectrum $C_{\ell}^{S Z}$ [63]. The priors on these primary cosmological parameters are listed in Table 2.

We adopt CMB data from WMAP5 64 66], as well as the observations at small angular scales including ACBAR 67, CBI 68, BOOMERANG 69 71] and QUAD 72. In addition, we adopt observational data of geometrical distances of the late Universe, including the Union data set of SN [76], the measurement of BAO scales in galaxy power spectra [77]\#8 and the $\mathrm{SH}_{0} \mathrm{ES}$ measurement of the Hubble constant $H_{0}=74.2 \pm 3.6$ [78]. A

\#5 A similar division of models can also be found in [30].

\#6 The upper bound $10^{-4}$ for $\epsilon_{*}$ is chosen so that these models cover inflation scenarios where detection of primordial B-mode would be difficult with CMB observations in the near future.

\#7 This is very near a fiducial wave number $k_{*}=0.017 \mathrm{Mpc}^{-1}$, which are suggested to be optimal for constraining the primordial power spectra from current data 61. Choice of fiducial wave number is also discussed in 23 .

\#8 We also performed the same analysis by adopting the data of halo power spectra from the catalogue of the SDSS Luminous Red Galaxies [73] instead of the BAO data. However, as long as combined with 


\begin{tabular}{l|c}
\hline \hline models & description \\
\hline$M_{\mathrm{HZ}}$ & HZ spectra \\
\hline$M_{n_{s}}$ & $0.8<n_{s}<1.2, r=\alpha_{s}=0$ \\
$M_{n_{s} r}$ & $0.8<n_{s}<1.2,0<r<0.5, \alpha_{s}=0$ \\
$M_{n_{s} \alpha_{s}}$ & $0.8<n_{s}<1.2, r=0,-0.1<\alpha_{s}<0.1$ \\
\hline$M_{\epsilon}$ & $0<\epsilon_{*}<0.1, \eta_{*}=\xi_{*}=0$ \\
$M_{\eta}$ & $0<\epsilon_{*}<10^{-4},-0.1<\eta_{*}<0.1, \xi_{*}=0$ \\
$M_{\epsilon \eta}$ & $0<\epsilon_{*}<0.1,-0.1<\eta_{*}<0.1, \xi_{*}=0$ \\
\hline \hline
\end{tabular}

Table 1: Models adopted in the analysis. $M_{\mathrm{HZ}}$ is the reference model that has HZ primordial power spectrum. Other models cover a model space for single-field slow-roll inflation. $M_{n_{s}}, M_{n_{s} r}, M_{n_{s} \alpha_{s}}$ have top-hat priors on the standard parameters of the primordial power spectra, and $M_{\epsilon}, M_{\eta}, M_{\epsilon \eta}$ have ones on the HSR parameters. Prior ranges are described in the last column. Regarding priors on $A_{s}$, we adopt a common prior $2.5<\ln \left[10^{10} A_{s}\right]<3.5$.

\begin{tabular}{l|c}
\hline \hline parameters & prior ranges \\
\hline$\omega_{b}$ & {$[0.02,0.025]$} \\
$\omega_{c}$ & {$[0.08,0.14]$} \\
$\theta_{s}$ & {$[1.02,1.06]$} \\
$\tau$ & {$[0.01,0.2]$} \\
$A_{S Z}$ & {$[0,4]$} \\
\hline \hline
\end{tabular}

Table 2: Top-hat priors on cosmological parameters, other than parameters of the primordial power spectra.

default data set that we conduct our analysis with is a combination of all the data above, which we denote as 'ALL'. On the other hand, we also make use of several other data sets, in order to assure ourselves that the results are not dragged by some extreme data. Adopted are data sets of WMAP5 alone and the combinations of WMAP5 with either the other $\mathrm{CMB}$ data at small angular scales, BAO, SN, or the $H_{0}$ measurement, which we denote as $+\mathrm{CMB},+\mathrm{BAO},+\mathrm{SN},+H_{0}$, respectively.

Computation of Bayes evidence is implemented with MultiNest [79], which is integrated in the Markov chain Monte Carlo (MCMC) sampling code CosmoMC [80] but uses the nested sampling [81] in stead of the MCMC sampling (See also Ref. [82]). Given a model

data of CMB, SN and the $H_{0}$ measurement, the results are very similar to those from the BAO data, which is also consistently observed in Ref. 74]. We suppose that this is due to the marginalization over scale-dependent galaxy biases. Although the matter power spectrum itself is a promising probe for the primordial power spectrum at small scales (See also [75] for a recent result), there are some difficulties in modeling nonlinear evolution of matter perturbations, biases of tracers, or effects of baryonic physics. For the time being, we do not adopt data of the matter power spectrum. 
$M$, MultiNest provides chains of samples from the posterior distribution $P(\Theta \mid$ data, $M)$ and Bayes evidence $E(M)$. Convergence is diagnosed by applying the Gelman-Rubin test with two independent chains. Typically $R-1<0.01$ is achieved.

\subsection{Standard parameterization}

In Table 3 we list Bayes factors for models with standard parameterization of the primordial power spectra. $M_{n_{s}}, M_{n_{s} r}$ and $M_{n_{s} \alpha_{s}}$ against a reference model $M_{\mathrm{HZ}}$. What we may first see in the table is that almost all data sets give positive Bayes factors. Although there are a few exceptions given by the $+H_{0}$ data set, they are by no means significant. This result shows us that current data negatively support a model with the HZ spectrum. In particular, the strongest negative support is brought from the ALL data set. Although Bayes factors depend on models we compare with $M_{\mathrm{HZ}}$, all models we adopt, $M_{n_{s}}, M_{n_{s} r}$ and $M_{n_{s} \alpha_{s}}$, give Bayes factors larger than 2.5. On Jeffreys' scale, these results correspond to strong negative evidences for $M_{\mathrm{HZ}}$. Such negative preference for the HZ power spectra was reported by many previous studies [36, 38, 40, 42, 44] and our result is consistent with the other recent result from WMAP5 [48]. However, significance in our result is considerably large as very recent observations are adopted. In Figure 1, we plotted the $1 \mathrm{~d}$ and $2 \mathrm{~d}$ marginalized posterior distributions of the parameters $n_{s}, r$, and $\alpha_{s}$ from the ALL data set. Clearly, the negative support for $M_{\mathrm{HZ}}$ originates from a poor fit of the HZ spectrum to the data, as the posterior distributions of $n_{s}$ for models $M_{n_{s}}, M_{n_{s} r}$ and $M_{n_{s} \alpha_{s}}$ consistently show preference for $n_{s} \neq 1$.

The Bayes evidences in Table 3 also show that the current data can be well-modeled by scalar perturbation with power-law power spectrum without the tensor mode (i.e. $M_{n_{s}}$ ). Let us regard $M_{n_{s}}$ as a base model and ALL as a base data set. When we include the tensor perturbation by varying $r>0$, the Bayes factors decrease from 4.3 for $M_{n_{s}}$ to 2.6 for $M_{n_{s} r}$. This suggests that current data negatively support presence of the tensor perturbation. In fact, the posterior distribution for $r$ peaks at $r=0$, as seen in Figure 1 , On the other hand, when we include the running of the scalar spectral index, the Bayes factor is almost unchanged from 4.3 for $M_{n_{s}}$ to 4.4 for $M_{n_{s} \alpha_{s}}$. This shows that even though the posterior distribution of $\alpha_{s}$ in Figure 1 peaks away from $\alpha_{s}=0$, the likelihood does not improve much from $M_{n_{s}}$ in most region of $\alpha_{s} \neq 0$ for the $M_{n_{s} \alpha_{s}}$ model. We conclude that presence of $\alpha_{s}$ is not disfavored by the data nor required in modeling the data.

From Table 3, the preference for $M_{n_{s}}$ over $M_{n_{s} r}$ is commonly observed with all the data sets. Also $M_{n_{s}}$ and $M_{n_{s} \alpha_{s}}$ are supported almost equally since the Bayesian evidences for these models do not differ by 1 at most. Totally, as $M_{n_{s}}$ is nested by $M_{n_{s} r}$ or $M_{n_{s} \alpha_{s}}$, we can safely conclude that $M_{n_{s}}$ best describes current data as far as we compare models with standard parameterization listed in Table 3 .

The constraints on cosmological parameters for these models are summarized in Table 4. As seen in the table, the current data can optimally be modeled by $M_{n_{s}}$. Therefore, we propose the constraint for $M_{n_{s}}$ as optimal one\#9. An optimal constraint on the pri-

\#9 We do not derive an optimal constraint by averaging over models, as our division of models, $M_{n_{s}}$, 


\begin{tabular}{crrr}
\hline \hline models & $M_{n_{s}}$ & $M_{n_{s} r}$ & $M_{n_{s} \alpha_{s}}$ \\
\hline WMAP5 & $+0.8 \pm 0.2$ & $-0.1 \pm 0.2$ & $+0.3 \pm 0.2$ \\
\hline+ CMB & $+1.4 \pm 0.2$ & $+0.5 \pm 0.2$ & $+1.2 \pm 0.2$ \\
\hline+ BAO & $+1.7 \pm 0.2$ & $+0.4 \pm 0.2$ & $+1.5 \pm 0.2$ \\
\hline+ SN & $+1.6 \pm 0.2$ & $+0.3 \pm 0.2$ & $+1.9 \pm 0.2$ \\
\hline$+H_{0}$ & $+0.5 \pm 0.2$ & $-0.2 \pm 0.2$ & $-0.4 \pm 0.2$ \\
\hline ALL & $+4.3 \pm 0.2$ & $+2.6 \pm 0.2$ & $+4.4 \pm 0.2$ \\
\hline \hline
\end{tabular}

Table 3: Bayes factors for models with the standard parameters of the primordial power spectra against the reference model $M_{\mathrm{HZ}}$.

mordial power spectra from the ALL data set is given by

$$
\begin{aligned}
\ln \left[10^{10} A_{s}\right] & =3.137_{-0.032}^{+0.028}, \\
n_{s} & =0.957_{-0.011}^{+0.010},
\end{aligned}
$$

where errors are given at $68 \%$ C.L. Regarding the tensor perturbation and the running of the scalar index, we find no evidence at present.

As a final remark of this section, let us comment on the choice of prior distributions and its relations to the resultant Bayes evidences. As we have mentioned in Section 3 , Bayes evidences depend on prior distributions. While a prior $0.8<n_{s}<1.2$ is often adopted in other studies [39, 44, priors $r<0.5$ and $\left|\alpha_{s}\right|<0.1$ may look moderately tight. More relaxed priors on $r$ and $\alpha_{s}$ may slightly decrease the Bayes evidences for $M_{n_{s} r}$ and $M_{n_{s} \alpha_{s}}$, which however hardly affects our conclusion.

\subsection{HSR parameterization}

In Tables 5 we present the Bayes factors for models with the HSR parameters, $M_{\epsilon}, M_{\eta}$ and $M_{\epsilon \eta}$, against $M_{\mathrm{HZ}}$. From the table, first we reconfirm that the $M_{\mathrm{HZ}}$ model is negatively supported from the data. With the ALL data set, the Bayes factors are 5.8, 4.7 and 4.2 for $M_{\epsilon}, M_{\eta}$ and $M_{\epsilon \eta}$, respectively. On Jeffreys' scale, these correspond to negative support for the model with the HZ spectrum at decisive or at least strong level. This shows that models of primordial power spectra with inflationary priors can quite well explain the data, while the data are very poorly fitted by the HZ spectrum.

Let us take a close look at the results so as to find plausible models of single-field slow-roll inflation from current data. In Figure 2, we presented the marginalized $1 \mathrm{~d}$ and $2 \mathrm{~d}$ posterior distributions of the HSR parameters $\epsilon_{*}$ and $\eta_{*}$. There we explicitly show the parameter region in $\epsilon_{*}-\eta_{*}$ plane that are removed by a prior $N>25$ for $\xi_{*}=0$. In Figure 3. we also present the marginalized posterior distributions of the amplitude of curvature power spectrum $A_{s}$ and the derived standard parameters, $n_{s}, r$ and $\alpha_{s}$, given as functions

$M_{n_{s} r}$ and $M_{n_{s} \alpha_{s}}$, is rather artificial, and not strongly based on theoretical motivations. 

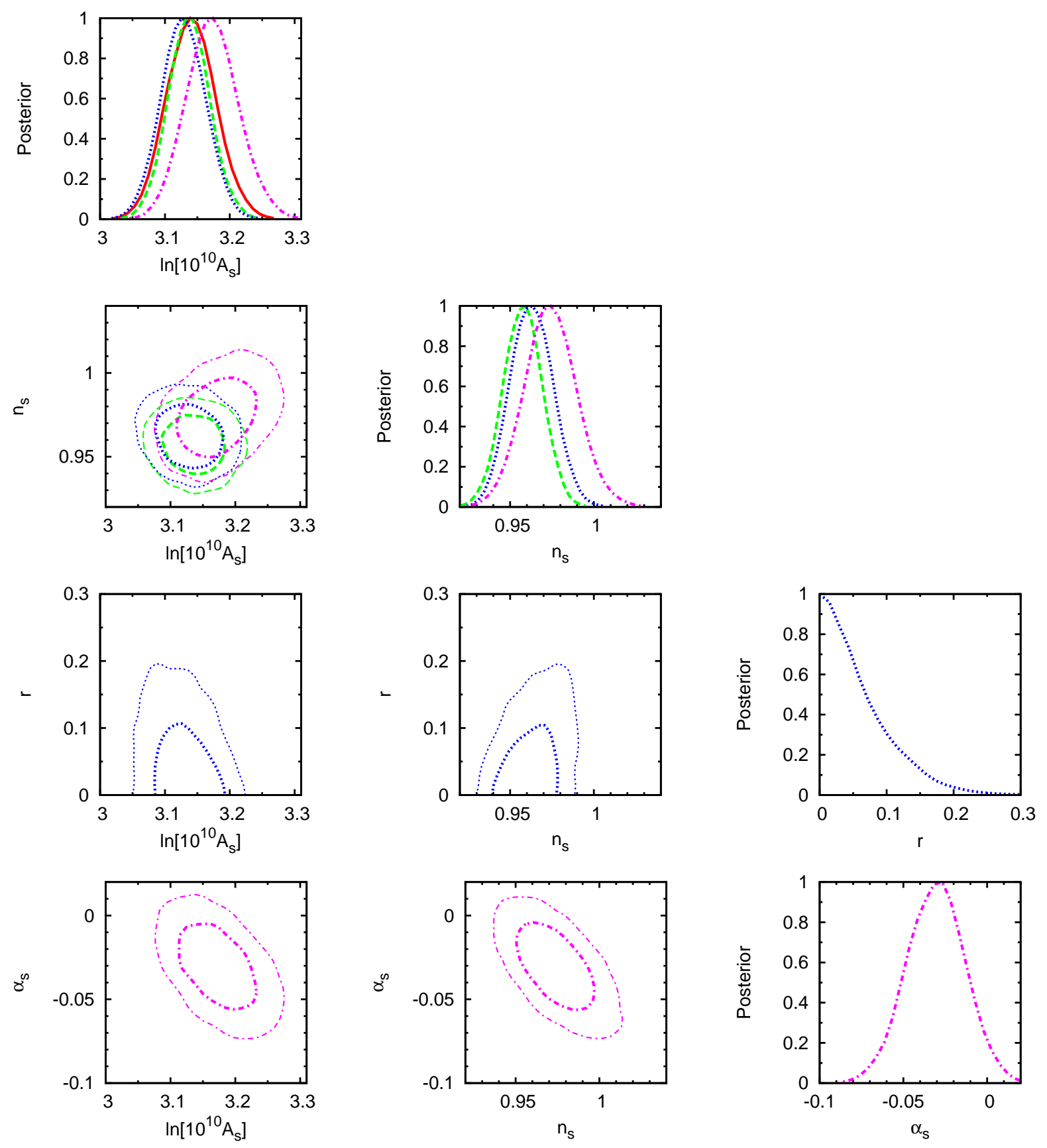

Figure 1: 1d and 2d marginalized posterior distributions of the standard parameters, $\log \left[10^{10} A_{s}\right], n_{s}, r$ and $\alpha_{s}$ from the ALL data set. Models shown are $M_{n_{s}}$ (dashed green line), $M_{n_{s} r}$ (dotted blue line), $M_{n_{s} \alpha_{s}}$ (dot-dashed magenta line) and the reference $M_{\mathrm{HZ}}$ (solid red line). 


\begin{tabular}{lrrr}
\hline \hline parameters & $M_{n_{s}}$ & $M_{n_{s} r}$ & $M_{n_{s} \alpha_{s}}$ \\
\hline$\omega_{b} \times 10^{2}$ & $2.263_{-0.041}^{+0.055}$ & $2.278_{-0.048}^{+0.051}$ & $2.227_{-0.059}^{+0.044}$ \\
$\omega_{c}$ & $0.1133_{-0.0032}^{+0.0028}$ & $0.1128_{-0.0033}^{+0.0027}$ & $0.1155_{-0.0036}^{+0.0032}$ \\
$\theta_{s} \times 10^{2}$ & $104.09_{-0.24}^{+0.19}$ & $104.10_{-0.19}^{+0.22}$ & $104.12_{-0.21}^{+0.21}$ \\
$\tau$ & $0.085_{-0.017}^{+0.013}$ & $0.084_{-0.015}^{+0.015}$ & $0.095_{-0.020}^{+0.015}$ \\
$\ln \left[10^{10} A_{s}\right]$ & $3.137_{-0.028}^{+0.028}$ & $3.128_{-0.030}^{+0.030}$ & $3.173_{-0.042}^{+0.034}$ \\
$n_{s}$ & $0.957_{-0.011}^{+0.010}$ & $0.962_{-0.011}^{+0.012}$ & $0.974_{-0.015}^{+0.013}$ \\
$r$ & - & $(0,0.15)$ & - \\
$\alpha_{s}$ & - & - & $-0.030_{-0.14}^{+0.18}$ \\
$n_{t}$ & - & $(-0.02,0)$ & - \\
\hline \hline
\end{tabular}

Table 4: Constraints for models with the standard parameters of the primordial power spectra, $M_{n_{s}}, M_{n_{s} r}$ and $M_{n_{s} \alpha_{s}}$, from the ALL data set. For bounded parameters, shown are mean values and $68 \%$ credible intervals. For unbounded parameters, 95\% credible intervals are only shown with parentheses.

of the HSR parameters (See Eqs. (16,18)). From Table 5 we consistently observe that the Bayes evidences for $M_{\epsilon}$ are larger than those for $M_{\eta}$ and $M_{\epsilon \eta}$, which are nearly equal, for all the data sets we adopted. With the ALL data set, the Bayes factor of $M_{\epsilon}$ against $M_{\eta}$ and $M_{\epsilon \eta}$ are 1.1 and 1.6. Thus the current data show slight preference for $M_{\epsilon}$ over $M_{\eta}$ and $M_{\epsilon \eta}$, which would be significant on Jeffreys' scale.

However, the preference for $M_{\epsilon}$ looks somewhat dependent on several assumptions we adopted in the analysis and the origin is not clear. One possible origin would be the prior $N>25$, for it eliminates the region $\epsilon_{*} \gtrsim 0.024$ if $\eta_{*}=0$, as can be seen from the shaded region in Figure 2. Thus in the models of $M_{\epsilon}$, the original top-hat prior $0<\epsilon_{*}<0.1$ is effectively substituted with a top-hat prior $0<\epsilon_{*}<0.024$ due to the prior $N>25$. As can be seen in the marginal posterior distribution of $\epsilon_{*}$ for $M_{\epsilon}$ in Figure 2, the effective prior region appears to give high likelihood, which results in boosting up the Bayesian evidence for $M_{\epsilon}$.

Let us examine the origin of preference for $M_{\epsilon}$ in more detail. We have repeated Monte Carlo analyses using the ALL data set, modifying the default setup in several different ways as follows:

(1) A prior $N>25$ is removed.

(2) Contribution from the tensor perturbation is omitted in the power spectra of CMB anisotropies.

(3) The prior ranges for $\epsilon_{*}$ and $\eta_{*}$ are extended from $\epsilon_{*},\left|\eta_{*}\right|<0.1$ to $\epsilon_{*},\left|\eta_{*}\right|<0.2$.

(4) The higher order HSR parameter $\xi_{*}$ is included with a top-hat prior $-0.01<\xi_{*}<$ 0.01 . 
The results are summarized in Table 6. From the modification (1), we first note that the Bayes fractor for $M_{\epsilon}$ significantly decreases from 5.8 to 4.7 by removal of a prior $N>25$. The resultant Bayes evidences are very comparable between models $M_{\epsilon}$ and $M_{\eta}$, while more complicated $M_{\epsilon \eta}$ gives a smaller Bayes evidence.

On the other hand, the largest difference between a model with large $\epsilon_{*}\left(\right.$ e.g. $\left.M_{\epsilon}\right)$ and one with small $\epsilon_{*}$ (e.g. $M_{\eta}$ ) would be whether primordial tensor perturbation is generated or not. Therefore the preference for $M_{\epsilon}$ can also possibly be induced from observably large contribution of the tensor perturbation in CMB anisotropies. However, this possibility is not supported by the result from the modification (2). The Bayes factor for $M_{\epsilon}$ does not decrease by omission of the tensor contribution, or even comes to increase slightly from 6.0 to 6.3. This is also as expected from the discussions in Section 4.1, where the model $M_{n_{s} r}$ with nonzero $r$ is less supported, compared with $M_{n_{s}}$ with vanishing $r$. From Figure 3 we also note that the running of scalar spectral index for these models is too small to be observed with current data (See also constraints on $\alpha_{s}$ for $M_{n_{s} \alpha_{s}}$ in Figure 1). Therefore the preference for $M_{\epsilon}$ is not from the running of the scalar spectral index either.

With all above demonstrations, we conclude that the preference for $M_{\epsilon}$ originates from a theoretical prior $N>25$. Current data is not very constraining enough for us to distinguish models adopted in our analysis and future observations should be await.

We also examine the dependence of Bayes evidence on top-hat priors on $\epsilon_{*}$ and $\eta_{*}$. The modification (3) in Table 6 shows that doubling of prior ranges from $\epsilon_{*},\left|\eta_{*}\right|<0.1$ to $\epsilon_{*},\left|\eta_{*}\right|<0.2$ decreases the Bayes factors for $M_{\eta}, M_{\epsilon \eta}$ by 0.8 and 1.4, respectively. These decreases are almost as expected from the dependence of Bayes evidences on prior ranges, that are about $\ln 2 \simeq 0.7$ and $2 \ln 2 \simeq 1.4$ for $M_{\eta}$ and $M_{\epsilon \eta}$, respectively. Contrastively, the Bayes evidence for $M_{\epsilon}$ is unchanged by the modification (3) because the priors $N>25$ dominates over the original top-hat prior on $\epsilon_{*}$.

So far we restricted ourselves to models with the HSR parameters up to $\epsilon$ and $\eta$. Let us examine possible preference for models with higher order HSR parameters. We have included the third lowest order HSR parameter $\xi_{*}$ with a top-hat prior $-0.01<\xi_{*}<0.01$ in the modification (4). From Table 6 we observe that the Bayes evidences almost equal or decrease from those with vanishing $\xi_{*}$. Therefore current data show no preference for models with nonzero $\xi_{*}$, and probably models with further higher order HSR parameters, as well. Here we should remark that above conclusion for models with nonzero $\xi_{*}$ relies on a prior $N>25$. As explicitly shown in [23], positive $\xi_{*} \simeq \mathcal{O}(0.01)$ may be favored when we omit the prior $N>25$. As we have discussed in Section 2, even for such large $\xi_{*}$, a sufficient e-folding number $N>25$ can possibly be achieved by higher order HSR parameters or other subsequent inflations. However, such complication may make the models less appealing.

The constraints on cosmological parameters for the models with the HSR parameters are presented in Table 7. From the table, we see that constraints on the amplitude and the spectral index of the primordial curvature power spectrum, $A_{s}$ and $n_{s}$, are almost independent of priors on the HSR parameters (See also Figure 3). Moreover, these constraints are identical with those of Eqs. (27) 28) from the $M_{n_{s}}$ models. Therefore we conclude that 


\begin{tabular}{crrr}
\hline \hline models & $M_{\epsilon}$ & $M_{\eta}$ & $M_{\epsilon \eta}$ \\
\hline WMAP5 & $+2.0 \pm 0.2$ & $+0.9 \pm 0.2$ & $+0.6 \pm 0.2$ \\
\hline+ CMB & $+2.9 \pm 0.2$ & $+1.7 \pm 0.2$ & $+1.1 \pm 0.2$ \\
\hline$+\mathrm{BAO}$ & $+3.2 \pm 0.2$ & $+1.9 \pm 0.2$ & $+1.6 \pm 0.2$ \\
\hline$+\mathrm{SN}$ & $+3.0 \pm 0.2$ & $+1.9 \pm 0.2$ & $+1.7 \pm 0.2$ \\
\hline$+H_{0}$ & $+1.8 \pm 0.2$ & $+0.6 \pm 0.2$ & $+0.3 \pm 0.2$ \\
\hline ALL & $+5.8 \pm 0.2$ & $+4.7 \pm 0.2$ & $+4.2 \pm 0.2$ \\
\hline \hline
\end{tabular}

Table 5: Bayes factors for models with the HSR parameters against the reference model $M_{\mathrm{HZ}}$.

\begin{tabular}{lrrr}
\hline \hline models & $M_{\epsilon}$ & $M_{\eta}$ & $M_{\epsilon \eta}$ \\
\hline ALL & $+5.8 \pm 0.2$ & $+4.7 \pm 0.2$ & $+4.2 \pm 0.2$ \\
\hline$(1)$ & $+4.7 \pm 0.2$ & $+4.6 \pm 0.2$ & $+3.7 \pm 0.2$ \\
\hline$(2)$ & $+5.9 \pm 0.2$ & $+4.6 \pm 0.2$ & $+4.2 \pm 0.2$ \\
\hline$(3)$ & $+5.8 \pm 0.2$ & $+3.9 \pm 0.2$ & $+2.8 \pm 0.2$ \\
\hline$(4)$ & $+4.3 \pm 0.2$ & $+5.1 \pm 0.2$ & $+3.6 \pm 0.2$ \\
\hline \hline
\end{tabular}

Table 6: Bayes factors for models with the HSR prameters against the reference model $M_{\mathrm{HZ}}$. Several extensions from the default setting are investigated: (1) removal of a prior on e-folding number; (2) omission of the tensor perturbation; (3) imposition of extended tophat priors, $\epsilon_{*},\left|\eta_{*}\right|<0.2$; (4) inclusion of the third lowest order HSR parameter $-0.01<$ $\xi_{*}<0.01$.

the optimal constraint we have proposed in Section 4.1 is robust with little dependence on priors from different inflationary models. However, constraints on the tensor perturbations and the running of the spectral index are largely dependent on inflationary priors and their presence is not suggested from current data.

\section{$5 \quad$ Summary and future outlook}

We have investigated constraints on the primordial power spectra and comparison of singlefield slow-roll inflation models using data from recent observations of CMB combined with measurements of BAO, SN and $H_{0}$. By employing Bayesian model selection, we found that a model with the scale-invariant HZ spectrum is strongly disfavored from current data, in comparison with several simple models allowing scale-dependence of the power spectra. We have also proposed an optimal constraint on the primordial power spectra of Eqs. (27,28).

Adopting our somewhat artificial division of models for single-field slow-roll inflation, 

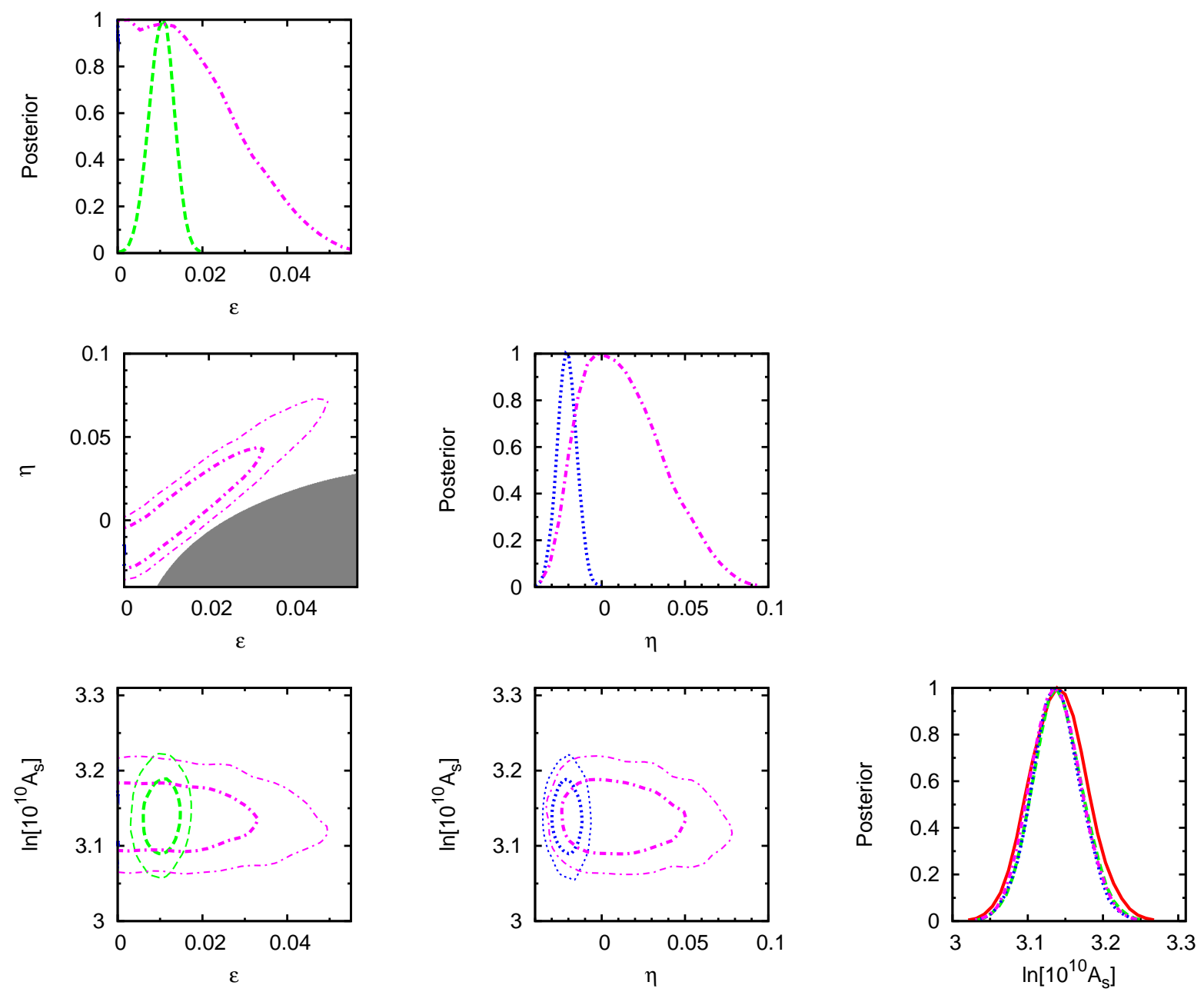

Figure 2: 1d and 2d marginalized posterior distributions for the HSR parameters, $\epsilon_{*}$ and $\eta_{*}$, and the amplitude of the curvature power spectrum $\ln \left[10^{10} A_{s}\right]$. Models shown are $M_{\epsilon}$ (dashed green line), $M_{\eta}$ (dotted blue line), $M_{\epsilon \eta}$ (dot-dashed magenta line) and the reference $M_{\mathrm{Hz}}$ (solid red line). The gray shaded region is excluded by a prior $N>25$ when $\xi_{*}=0$. 

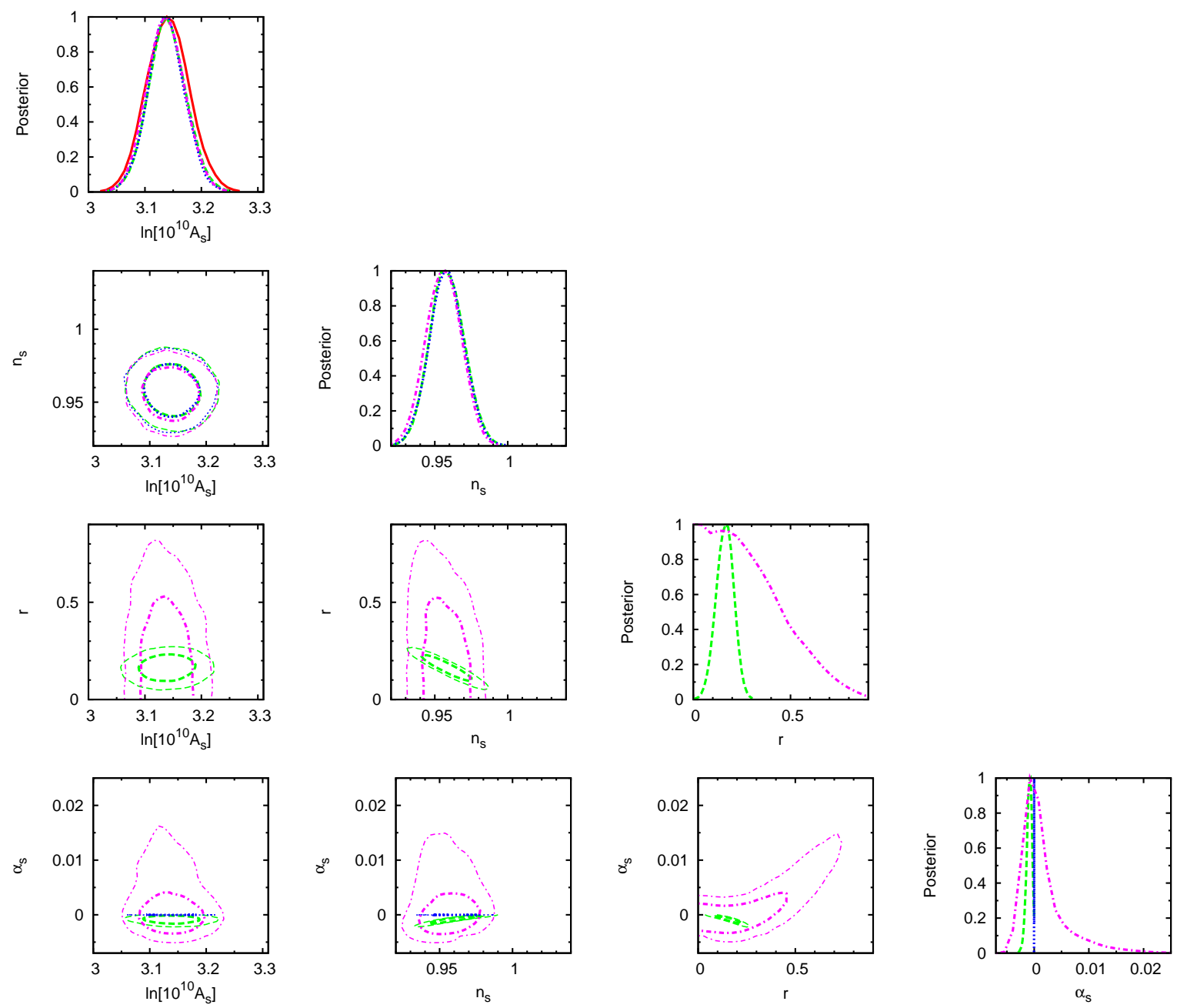

Figure 3: Same figure as in Figure 2 but the posterior distributions for the standard parameters, $\ln \left[10^{10} A_{s}\right], n_{s}, r$ and $\alpha_{s}$, are shown. 


\begin{tabular}{lrrr}
\hline \hline parameters & $M_{\epsilon}$ & $M_{\eta}$ & $M_{\epsilon \eta}$ \\
\hline$\omega_{b} \times 10^{2}$ & $2.261_{-0.048}^{+0.052}$ & $2.263_{-0.049}^{+0.048}$ & $2.260_{-0.050}^{+0.051}$ \\
$\omega_{c}$ & $0.1136_{-0.0031}^{+0.0031}$ & $0.1135_{-0.00232}^{+0.0029}$ & $0.1134_{-0.0292}^{+0.0029}$ \\
$\theta_{s} \times 10^{2}$ & $104.12_{-0.19}^{+0.22}$ & $104.13_{-0.20}^{+0.21}$ & $104.11_{-0.19}^{+0.23}$ \\
$\tau$ & $0.086_{-0.017}^{+0.013}$ & $0.085_{-0.014}^{+0.015}$ & $0.084_{-0.018}^{+0.012}$ \\
$\epsilon_{*}$ & $0.0104_{-0.0028}^{+0.0028}$ & - & $(0,0.039)$ \\
$\eta_{*}$ & - & $-0.0208_{-0.0051}^{+0.0059}$ & $0.013_{-0.030}^{+0.015}$ \\
$\ln \left[10^{10} A_{s}\right]$ & $3.137_{-0.032}^{+0.028}$ & $3.137_{-0.030}^{+0.030}$ & $3.137_{-0.036}^{+0.027}$ \\
\hline$n_{s}$ & $0.958_{-0.011}^{+0.011}$ & $0.958_{-0.010}^{+0.011}$ & $0.956_{-0.012}^{+0.011}$ \\
$r$ & $0.163_{-0.043}^{+0.044}$ & - & $(0,0.64)$ \\
$\alpha_{s} \times 10^{3}$ & $0.92_{-0.32}^{+0.57}$ & - & $1.57_{-3.20}^{+0.06}$ \\
$n_{t} \times 10^{2}$ & $-2.10_{-0.58}^{+0.58}$ & - & $(-8.1,0)$ \\
\hline \hline
\end{tabular}

Table 7: Constraints for models with the inflationary HSR parameters, $M_{\epsilon}, M_{\eta}$ and $M_{\epsilon \eta}$, from the ALL data set. For bounded parameters, shown are mean values and $68 \%$ credible intervals. For unbounded parameters, 95\% credible intervals are only shown with parentheses.

$M_{\epsilon}, M_{\eta}$ and $M_{\epsilon \eta}$, with a theoretical prior $N>25$, we have found the Bayes evidences for these models from current data are almost comparable, but there is a slight but consistent preference for $M_{\epsilon}$ over the others from various data sets. We have demonstrated several extension in order to identify the origin of the preference for $M_{\epsilon}$. A presence of the tensor perturbation is not signified and we have found that the preference originates from a prior $N>25$ which appears to make a prior ranges for $\epsilon_{*}$ center around the region of high likelihood. We have also found that higher order slow-roll parameters are not required from current data. Thus we conclude that while simple models of single-field slow-roll inflation can describe current cosmological observations, data is not enough constraining to distinguish these models we adopted.

Planck [83] and several other ground-based CMB surveys, such as QUIET [84], PolarBeaR [85], etc. are now under way. In the near future, the tensor perturbation will be probed down to $r \simeq 0.01$ by these surveys. This allow us to discriminate models of single-field inflation through the prediction on the primordial power spectra or the slowroll parameters. Indeed, one of our division of models, $M_{\epsilon}$, will be strongly refused, if the primordial tensor perturbation is not detected by those surveys. Given data from these future surveys, employment of Bayesian model selection would be of great help in several ways, such as constraining cosmological parameters in an optimal way, and assessing a support for a cosmological model statistically. 


\section{Acknowledgment}

T.S. would like to thank the Japan Society for the Promotion of Science for financial support. This work is supported by Grant-in-Aid for Scientific research from the Ministry of Education, Science, Sports, and Culture (MEXT), Japan, under Contract No. 14102004 (M.K.), and also by World Premier International Research Center Initiative, MEXT, Japan.

\section{References}

[1] A. A. Starobinsky, Phys. Lett. B 91 (1980) 99.

[2] A. H. Guth, Phys. Rev. D 23, 347 (1981).

[3] K. Sato, Mon. Not. Roy. Astron. Soc. 195, 467 (1981).

[4] A. D. Linde, Phys. Lett. B 108, 389 (1982).

[5] A. J. Albrecht and P. J. Steinhardt, Phys. Rev. Lett. 48, 1220 (1982).

[6] A. A. Starobinsky, JETP Lett. 30 (1979) 682 [Pisma Zh. Eksp. Teor. Fiz. 30 (1979) 719].

[7] V. F. Mukhanov and G. V. Chibisov, JETP Lett. 33 (1981) 532 [Pisma Zh. Eksp. Teor. Fiz. 33 (1981) 549].

[8] S. W. Hawking, Phys. Lett. B 115, 295 (1982).

[9] A. A. Starobinsky, Phys. Lett. B 117 (1982) 175.

[10] A. H. Guth and S. Y. Pi, Phys. Rev. Lett. 49, 1110 (1982).

[11] A. D. Linde, Phys. Lett. B 116, 335 (1982).

[12] J. M. Bardeen, P. J. Steinhardt and M. S. Turner, Phys. Rev. D 28, 679 (1983).

[13] L. F. Abbott and M. B. Wise, Nucl. Phys. B 244, 541 (1984).

[14] E. J. Copeland, E. W. Kolb, A. R. Liddle and J. E. Lidsey, Phys. Rev. D 48, 2529 (1993) arXiv:hep-ph/9303288.

[15] J. E. Lidsey, A. R. Liddle, E. W. Kolb, E. J. Copeland, T. Barreiro and M. Abney, Rev. Mod. Phys. 69, 373 (1997) arXiv:astro-ph/9508078.

[16] S. M. Leach and A. R. Liddle, Mon. Not. Roy. Astron. Soc. 341, 1151 (2003) arXiv:astro-ph/0207213. 
[17] R. Easther and W. H. Kinney, Phys. Rev. D 67, 043511 (2003) arXiv:astro-ph/0210345.

[18] W. H. Kinney, E. W. Kolb, A. Melchiorri and A. Riotto, Phys. Rev. D 69, 103516 (2004) arXiv:hep-ph/0305130].

[19] S. M. Leach and A. R. Liddle, Phys. Rev. D 68, 123508 (2003) arXiv:astro-ph/0306305].

[20] H. Peiris and R. Easther, JCAP 0607, 002 (2006) arXiv:astro-ph/0603587.

[21] W. H. Kinney, E. W. Kolb, A. Melchiorri and A. Riotto, Phys. Rev. D 74, 023502 (2006) arXiv:astro-ph/0605338].

[22] J. Martin and C. Ringeval, JCAP 0608, 009 (2006) [arXiv:astro-ph/0605367].

[23] H. Peiris and R. Easther, JCAP 0610, 017 (2006) arXiv:astro-ph/0609003.

[24] F. Finelli, M. Rianna and N. Mandolesi, JCAP 0612, $006 \quad$ (2006) arXiv:astro-ph/0608277.

[25] J. Lesgourgues and W. Valkenburg, Phys. Rev. D 75, 123519 (2007) arXiv:astro-ph/0703625.

[26] B. A. Powell and W. H. Kinney, JCAP 0708, 006 (2007) [arXiv:0706.1982 [astro-ph]].

[27] J. Lesgourgues, A. A. Starobinsky and W. Valkenburg, JCAP 0801, 010 (2008) arXiv:0710.1630 [astro-ph]].

[28] R. Bean, D. J. H. Chung and G. Geshnizjani, Phys. Rev. D 78, 023517 (2008) arXiv:0801.0742 [astro-ph]].

[29] J. Hamann, J. Lesgourgues and W. Valkenburg, JCAP 0804, 016 (2008) arXiv:0802.0505 [astro-ph]].

[30] P. Adshead and R. Easther, JCAP 0810, 047 (2008) [arXiv:0802.3898 [astro-ph]].

[31] N. Agarwal and R. Bean, Phys. Rev. D 79, 023503 (2009) [arXiv:0809.2798 [astro-ph]].

[32] B. A. Powell, K. Tzirakis and W. H. Kinney, JCAP 0904, 019 (2009) arXiv:0812.1797 [astro-ph]].

[33] E. Komatsu et al. [WMAP Collaboration], Astrophys. J. Suppl. 180, 330 (2009) arXiv:0803.0547 [astro-ph]].

[34] A. Slosar et al., Mon. Not. Roy. Astron. Soc. 341, L29 (2003) arXiv:astro-ph/0212497. 
[35] M. Beltran, J. Garcia-Bellido, J. Lesgourgues, A. R. Liddle and A. Slosar, Phys. Rev. D 71, 063532 (2005) arXiv:astro-ph/0501477.

[36] R. Trotta, Mon. Not. Roy. Astron. Soc. 378, 72 (2007) arXiv:astro-ph/0504022.

[37] M. Bridges, A. N. Lasenby and M. P. Hobson, Mon. Not. Roy. Astron. Soc. 369, 1123 (2006) arXiv:astro-ph/0511573.

[38] M. Kunz, R. Trotta and D. Parkinson, Phys. Rev. D 74, 023503 (2006) arXiv:astro-ph/0602378.

[39] D. Parkinson, P. Mukherjee and A. R. Liddle, Phys. Rev. D 73, 123523 (2006) arXiv:astro-ph/0605003.

[40] M. Bridges, A. N. Lasenby and M. P. Hobson, arXiv:astro-ph/0607404.

[41] C. Pahud, A. R. Liddle, P. Mukherjee and D. Parkinson, Phys. Rev. D 73, 123524 (2006) arXiv:astro-ph/0605004].

[42] A. R. Liddle, P. Mukherjee and D. Parkinson, arXiv:astro-ph/0608184.

[43] A. F. Heavens, T. D. Kitching and L. Verde, Mon. Not. Roy. Astron. Soc. 380, 1029 (2007) arXiv:astro-ph/0703191.

[44] C. Gordon and R. Trotta, Mon. Not. Roy. Astron. Soc. 382, 1859 (2007) arXiv:0706.3014 [astro-ph]].

[45] P. Mukherjee and A. R. Liddle, arXiv:0803.1738 [astro-ph].

[46] R. Trotta, Contemp. Phys. 49, 71 (2008) [arXiv:0803.4089 [astro-ph]].

[47] P. Mukherjee and D. Parkinson, Int. J. Mod. Phys. A 23 (2008) 787.

[48] M. Bridges, F. Feroz, M. P. Hobson and A. N. Lasenby, arXiv:0812.3541 [astro-ph].

[49] A. R. Liddle, arXiv:0903.4210 [hep-th].

[50] I. Sollom, A. Challinor and M. P. Hobson, Phys. Rev. D 79, 123521 (2009) arXiv:0903.5257 [astro-ph.CO]].

[51] K. Ichikawa, M. Kawasaki, K. Nakayama, T. Sekiguchi and T. Takahashi, JCAP 0908, 013 (2009) [arXiv:0905.2237 [astro-ph.CO]].

[52] J. Valiviita and T. Giannantonio, arXiv:0909.5190 [astro-ph.CO].

[53] G. Ballesteros, J. A. Casas, J. R. Espinosa, R. Ruiz de Austri and R. Trotta, JCAP 0803, 018 (2008) [arXiv:0711.3436 [hep-ph]]. 
[54] D. Baumann et al. [CMBPol Study Team Collaboration], AIP Conf. Proc. 1141, 10 (2009) arXiv:0811.3919 [astro-ph]].

[55] S. M. Leach, A. R. Liddle, J. Martin and D. J. Schwarz, Phys. Rev. D 66, 023515 (2002) arXiv:astro-ph/0202094.

[56] M. B. Hoffman and M. S. Turner, Phys. Rev. D 64, 023506 (2001) arXiv:astro-ph/0006321.

[57] W. H. Kinney, Phys. Rev. D 66, 083508 (2002) arXiv:astro-ph/0206032.

[58] A. R. Liddle, Phys. Rev. D 68, 103504 (2003) arXiv:astro-ph/0307286].

[59] E. D. Stewart and D. H. Lyth, Phys. Lett. B 302, 171 (1993) arXiv:gr-qc/9302019.

[60] H. V. Peiris and R. Easther, JCAP 0807, 024 (2008) arXiv:0805.2154 [astro-ph]].

[61] M. Cortes, A. R. Liddle and P. Mukherjee, Phys. Rev. D 75, 083520 (2007) arXiv:astro-ph/0702170.

[62] A. Kosowsky, M. Milosavljevic and R. Jimenez, Phys. Rev. D 66, 063007 (2002) arXiv:astro-ph/0206014.

[63] E. Komatsu and U. Seljak, Mon. Not. Roy. Astron. Soc. 336, 1256 (2002) arXiv:astro-ph/0205468.

[64] J. Dunkley et al. [WMAP Collaboration], Astrophys. J. Suppl. 180, 306 (2009) arXiv:0803.0586 [astro-ph]].

[65] M. R. Nolta et al. [WMAP Collaboration], Astrophys. J. Suppl. 180, 296 (2009) arXiv:0803.0593 [astro-ph]].

[66] G. Hinshaw et al. [WMAP Collaboration], Astrophys. J. Suppl. 180, 225 (2009) arXiv:0803.0732 [astro-ph]].

[67] C. L. Reichardt et al., arXiv:0801.1491 [astro-ph].

[68] J. L. Sievers et al., Astrophys. J. 660, 976 (2007) arXiv:astro-ph/0509203.

[69] W. C. Jones et al., Astrophys. J. 647, 823 (2006) arXiv:astro-ph/0507494.

[70] F. Piacentini et al., Astrophys. J. 647, 833 (2006) arXiv:astro-ph/0507507.

[71] T. E. Montroy et al., Astrophys. J. 647, 813 (2006) arXiv:astro-ph/0507514.

[72] R. B. Friedman et al. [QUaD collaboration], arXiv:0901.4334 [astro-ph.CO].

[73] B. A. Reid et al., arXiv:0907.1659 [astro-ph.CO]. 
[74] F. Finelli, J. Hamann, S. M. Leach and J. Lesgourgues, arXiv:0912.0522 [astroph.CO].

[75] H. V. Peiris and L. Verde, arXiv:0912.0268 [astro-ph.CO].

[76] M. Kowalski et al. [Supernova Cosmology Project Collaboration], Astrophys. J. 686, 749 (2008) arXiv:0804.4142 [astro-ph]].

[77] W. J. Percival et al., arXiv:0907.1660 [astro-ph.CO].

[78] A. G. Riess et al., arXiv:0905.0695 [astro-ph.CO].

[79] F. Feroz, M. P. Hobson and M. Bridges, arXiv:0809.3437 [astro-ph].

[80] A. Lewis and S. Bridle, Phys. Rev. D 66, 103511 (2002) [arXiv:astro-ph/0205436].

[81] Skilling J., 2004, AIP Conference Proceedings of the 24th International Workshop on Bayesian Inference and Maximum Entropy Methods in Science and Engineering, Vol. 735 , pp. 395-405

[82] P. Mukherjee, D. Parkinson and A. R. Liddle, Astrophys. J. 638, L51 (2006) arXiv:astro-ph/0508461.

[83] [Planck Collaboration], arXiv:astro-ph/0604069.

[84] D. Samtleben and f. t. Q. Collaboration, Nuovo Cim. 122B, 1353 (2007) arXiv:0802.2657 [astro-ph]].

[85] http://bolo.berkeley.edu/polarbear/ 